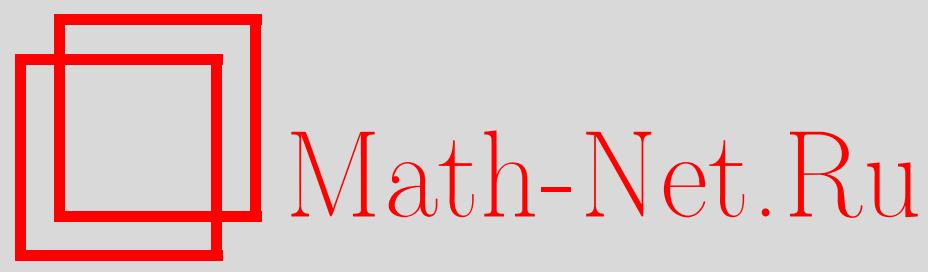

С. Ю. Оревков, Сильная положительность в правоинвариантном порядке на группе кос и квазиположительность, Матем. заметки, 2000, том 68, выпуск 5, 692-698

DOI: https://doi.org/10.4213/mzm990

Использование Общероссийского математического портала Math-Net.Ru подразумевает, что вы прочитали и согласны с пользовательским соглашением http://www.mathnet.ru/rus/agreement

Параметры загрузки:

IP : 54.84 .234 .179

26 апреля 2023 г., 13:48:27

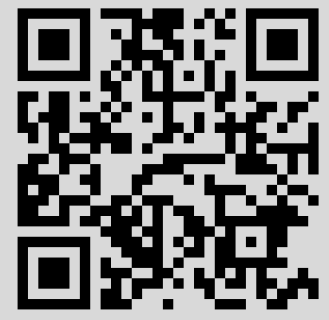




\title{
СИЛЬНАЯ ПОЛОЖИТЕЛЬНОСТЬ В ПРАВО-ИНВАРИАНТНОМ ПОРЯДКЕ НА ГРУППЕ КОС И КВАЗИПОЛОЖИТЕЛЬНОСТЬ
}

\author{
С. Ю. Оревков
}

Деорнуапостроил право-инвариантный порядок на группе кос $B_{n}$, однозначно определяемый тем, что $\beta_{0} \sigma_{i} \beta_{1}>1$, если $\beta_{0}, \beta_{1}$ - слова, составленные из $\sigma_{i+1}^{ \pm 1}, \ldots, \sigma_{n-1}^{ \pm 1}$. Коса называется сильно положительной, если $\alpha \beta \alpha^{-1}>1$ для всех $\alpha \in B_{n}$. В заметке доказано, что коса $\beta_{0}\left(\sigma_{1} \sigma_{2} \ldots \sigma_{n-1}\right)\left(\sigma_{n-1} \sigma_{n-2} \ldots \sigma_{1}\right)$ сильно положительна, если слово $\beta_{0}$ не содержит $\sigma_{1}^{ \pm 1}$. Дано также геометрическое доказательство результата Баркера и Лавера о том, что стандартные образующие группы кос сильно положительны. Наконец, обсуждается связь право-инвариантного порядка с квазиположительностью.

Библиограффия: 10 названий.

Пусть $B_{n}-$ группа кос из $n$ нитей:

$$
\left.B_{n}=\left\langle\sigma_{1}, \ldots, \sigma_{n-1}\right| \sigma_{i} \sigma_{j}=\sigma_{j} \sigma_{i} \text { при }|i-j|>1, \sigma_{i} \sigma_{j} \sigma_{i}=\sigma_{j} \sigma_{i} \sigma_{j} \text { при }|i-j|=1\right\rangle .
$$

Деорнуа [1]-[3] доказал, что существует полньй право-инвариантньй порядок на элементах группы $B_{n}$ (право-инвариантньй значит $\alpha<\beta \Rightarrow \alpha \gamma<\beta \gamma \forall \alpha, \beta, \gamma$ ), однозначно определяемьй условием

$$
\beta_{0} \sigma_{i} \beta_{1}>1 \text {, если } \beta_{0}, \beta_{1}-\text { слова, составленные из } \sigma_{i+1}^{ \pm 1}, \ldots, \sigma_{n-1}^{ \pm 1} \text {. }
$$

Он дал также эффективный на практике алгоритм, определяющий, является ли данная коса положительной, тривиальной или отрицательной (коса $\beta$ назьвается поло жительной, если $\beta>1$ ). Недавно авторы статьи [4] дали красивую геометрическую интерпретацию право-инвариантного порядка (см. п. 2 ниже) и другие алгоритмы сравнения кос, про один из которых доказана квадратичность по времени.

Будем говорить, что коса $\beta \in B_{n}$ сильно положительна, если $\alpha \beta \alpha^{-1}>1$ для любого $\alpha \in B_{n}$. Ясно, что сильно положительные косы образуют полугрупшу, инвариантную относительно сопряжения. Коса $\beta=\sigma_{1}^{2} \sigma_{2}^{-2}$ является примером положительной, но не сильно положительной косы, потому что $\alpha \beta \alpha^{-1}<1$ для $\alpha=\sigma_{2} \sigma_{1}^{2} \sigma_{2}$ (см. [4]). В настоящей заметке мы обсуждаем некоторые классы сильно положительных кос.

ТЕорема А (Баркел [5], Лавер [6]). Образующие $\sigma_{k}$ сильно положительнь.

Доказательства, приведенные в [5], [6], - алгебраические. Недавно Вист [7] дал геометрическое доказательство, основанное на идеях и результатах статьи [4]. В п. 3 мы

Автор благодарит институт им. Макса Планка за финансовую поддержку и гостеприимство. 
даем еще одно доказательство. В нем мы получаем теорему А как непосредственное следствие из основного результата работы [4].

Коса $\beta \in B_{n}$ называется квазиположительной, если $\beta=\left(\gamma_{1} \sigma_{1} \gamma_{1}^{-1}\right) \cdots\left(\gamma_{s} \sigma_{1} \gamma_{s}^{-1}\right)$ при некоторых $\gamma_{1}, \ldots, \gamma_{s} \in B_{n}$. Это понятие было введено Рудольфом [8], [9]; он показал, что коса квазиположительна тогда и только тогда, когда она является граничной косой алгебраической функции $w(z)$, неявно заданной в диске уравнением $w^{n}+$ $a_{1}(z) w^{n-1}+\cdots+a_{n}(z)=0, a_{i} \in \mathbb{C}[z]$. Вопрос о квазиположительности косы естественно возникает в топологии плоских вещественных алгебраических кривых [10]. Алгоритм распознавания квазиположительных кос известен лишь при $n=3$. Некоторые необходимые условия для квазиположительности имеются в [9], [10]. Теорема А дает еще одно необходимое условие для квазиположительности косы, поскольку она, очевидно, эквивалентна утверждению о том, что квазиположительная коса положительна в право-инвариантном порядке.

Обозначим через $Q_{n}$ (соответственно $P_{n}$ ) множество квазиположительных (соответственно сильно положительных) кос в $B_{n}$. В этих обозначениях теорему А можно переформулировать как $Q_{n} \subset P_{n}$. Обозначим

$$
\pi_{n}:=\left(\sigma_{1} \sigma_{2} \ldots \sigma_{n-1}\right) \cdot\left(\sigma_{n-1} \sigma_{n-2} \ldots \sigma_{1}\right) \in B_{n}
$$

ТЕоремА В. Если $\beta_{0}-$ слово, составленное из $\sigma_{2}^{ \pm 1}, \ldots, \sigma_{n-1}^{ \pm 1}$, то коса $\beta=\beta_{0} \pi_{n}$ сильно положстельна.

Это показьвает, что $P_{n} \neq Q_{n}$. Действительно, для косы $\beta=\prod_{j} \sigma_{i_{j}}^{k_{j}}$ обозначим $e(\beta)=\sum_{j} k_{j}$. Тогда теорема В влечет

СлЕДСТВИЕ. Если $\beta_{0}-$ слово, составленное из $\sigma_{2}^{ \pm 1}, \ldots, \sigma_{n-1}^{ \pm 1}, u e\left(\beta_{0}\right)<2-2 n$ (например $\beta_{0}=\sigma_{2}^{-2 n}$ ), то $\beta_{0} \pi_{n} \in P_{n} \backslash Q_{n}$.

Пусть $\beta \in B_{n}$ - коса и $L-$ соответствующее зацепление (замькание косы $\beta$ ). Пусть $L_{1}, \ldots, L_{k}-$ компоненты $L$. Пусть $n_{1}, \ldots, n_{k}$ - любые положительные целые числа и $\beta_{1}, \ldots, \beta_{k}$ - любые косы, $\beta_{j} \in B_{n_{j}}$. Тогда $\left(\beta_{1}, \ldots, \beta_{k}\right)$-обмотка косы $\beta$ определяется как коса, для которой соответствующее зацепление получено из $L$ заменой каждого $L_{j}$ на зацепление в трубчатой окрестности зацепления $L_{j}$, задаваемое косой $\beta_{j}$. Например, коса $\beta$ из теоремы В есть $\left(\beta_{0}, 1\right)$-обмотка косы $\sigma_{1}^{2} \in B_{2}$. Будем говорить, что коса $i$-положительна, если она является произведением кос вида $\beta_{0} \sigma_{i} \beta_{1}$, где $\beta_{0}, \beta_{1}-$ слова, составленные из $\sigma_{i+1}^{ \pm 1}, \ldots, \sigma_{n-1}^{ \pm 1}$. Тогда представляется правдоподобным, что теорема В есть частный случай следующего более общего факта.

ГИПоТЕЗА. Если любая коса, сопряженная косе $\beta, 1$-полохительна и $\beta^{\prime}$ - обмотка косы $\beta$, то любая коса, сопряжсеная косе $\beta^{\prime}$, 1-положительна.

1. Доказательство теоремы В. Пусть $D \subset \mathbb{C}$ - замкнутый единичньй диск и $X_{n}=\left\{x_{0}, \ldots, x_{n+1}\right\}$, где $-1=x_{0}<x_{1}<\cdots<x_{n}<x_{n+1}=1$ - вещественные в $D$. Геометрическая коса - это набор из $n$ гладких непересекающихся кривых (называемых нитя.ми) в цилиндре $D \times[0,1]$ таких, что проекция каждой нити на $[0,1]$ есть диффеоморфизм и множество концов всех нитей совпадает с $X_{n} \times\{0,1\}$. Косой назьвается класс эквивалентности геометрических кос относительно изотопий цилиндра $D \times[0,1]$, неподвижных на $D \times\{0,1\}$. 
Пусть $p$ - проекция $(z, t) \mapsto(\operatorname{Re} z, t)$. Геометрическая коса находится $в$ общем положении, если проекции нитей трансверсальны между собой и $t$-координаты их пересечений различны. Стандартная образующая $\sigma_{i} \in B_{n}$ и обратный к ней элемент изображены на рис. 1 . Рисунок для $\alpha \cdot \beta$, где $\alpha, \beta \in B_{n}$, получается, если рисунок косы $\beta$ приставить сверху к рисунку косы $\alpha$. Таким образом, геометрическая коса $B$ в общем положении определяет слово из стандартных образующих $\sigma_{1}^{ \pm 1}, \ldots, \sigma_{n-1}^{ \pm 1}$, в котором каждая буква отвечает двойной точке множества $p(B)$.

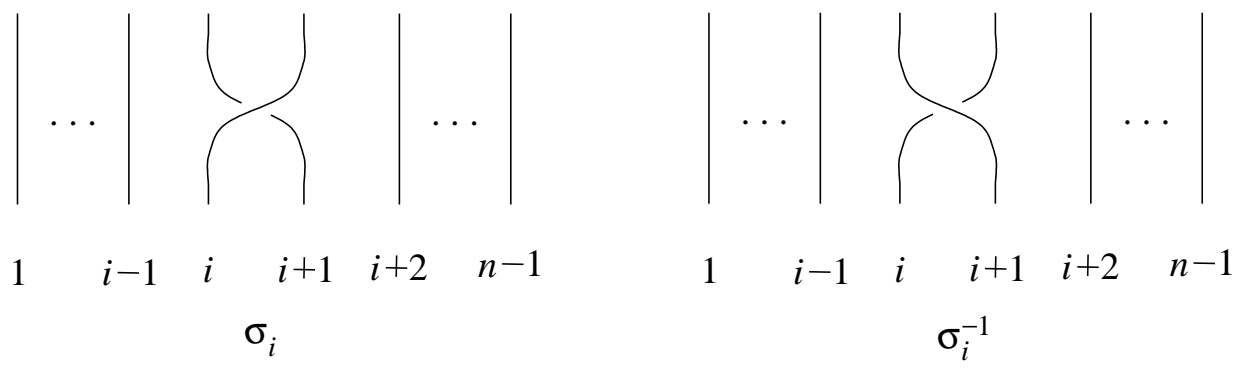

Рис. 1

Пусть $\beta_{0}, \beta$ - косы из теоремы В. Тогда геометрическая коса $B$, представляющая косу $\beta$, обладает следующим свойством. Существует гладкая поверхность $C \subset(D \times$ $[0,1]) \backslash B$, расслоенная на гладкие окружности $C_{t} \subset \operatorname{Int} D \times\{t\}, t \in[0,1]$, такая, что точки $x_{2}, \ldots, x_{n}$ лежат внутри $C_{0}$, точка $x_{1}$ лежит вне $C_{0}$ и окружность $C_{0}$ отображается на $C_{1}$ при диффеоморфизме $D \times\{0\} \rightarrow D \times\{1\},(z, 0) \mapsto(z, 1)$.

Обозначим через $T$ полноторие, полученное из $D \times[0,1]$ отождествлением $D \times\{0\}$ и $D \times\{1\}$. Мы будем одинаково обозначать подмножества в $D \times[0,1]$ и их образы в $T$. Пусть $S$ - компонента геометрической косы $B$, соответствующая первой нити, т.е. компонента, проходящая через точку $\left(x_{1}, 0\right)$. Тогда образ поверхности $C$ в $T$ есть замкнутая поверхность, гомеоморфная тору, охватьвающая $B \backslash S$, причем $S$ имеет индекс зацепления +1 с тором $C$ в следующем смысле. Пусть $A \subset T$ - кольцо такое, что одна компонента края $\partial A$ изтопна на $\partial T$ отрезку $y \times[0,1], y \in \partial D$, а другая компонента края $\partial A$ лежит на $C$. Тогда индекс зацепления $S$ и $C$ определяется как индекс пересечения $S$ и $A$ (здесь и далее мы оставляем читателю заботиться об ориентациях).

Пусть $\alpha$-любая коса, и положим $\beta^{\prime}=\alpha \beta \alpha^{-1}$. Нам надо доказать, что $\beta^{\prime}>1$ в право-инвариантном порядке. Согласно результатам Деорнуа [1]-[3] каноническая форма любой неположительной косы - это слово из $\sigma_{1}^{ \pm 1}, \ldots, \sigma_{n-1}^{ \pm 1}$, не содержащее $\sigma_{1}^{+1}$. Таким образом, чтобы доказать, что $\beta^{\prime}>1$, достаточно убедиться в том, что каждое слово, представляющее $\gamma$, содержит $\sigma_{1}^{+1}$. Рассмотрим произвольное слово $w$, представляющее $\beta^{\prime}$, и пусть $B^{\prime}$ - соответствующая геометрическая коса в общем положении. $B^{\prime}$ получена из $B$ изотопией полнотория $T$, так как $\beta^{\prime}$ и $\beta$ сопряжены. Обозначим через $C^{\prime}$ и $S^{\prime}$ образы $C$ и $S$ при этой изотопии. Индекс зацепления не меняется при изотопиях, поэтому индекс зацепления $S^{\prime}$ и $C^{\prime}$ в вышеуказанном смысле равен +1 .

Будем предполагать, что поверхность $C^{\prime}$ минимальна: мы представляем себе это так, что $B^{\prime}$ сделано из жесткой проволоки, а $C^{\prime}$ - резиновая пленка, отделяющая $S^{\prime}$ от $B^{\prime} \backslash S^{\prime}$. Более формально это означает следующее. При $t \in[0,1]$ обозначим через $D_{t}$ диск $D \times\{t\}$, и пусть $B_{t}^{\prime}$ (соответственно $S_{t}^{\prime}, C_{t}^{\prime}$ ) - пересечение $B^{\prime}$ (соответственно $S^{\prime}, C^{\prime}$ ) 
с $D_{t}$. После того, как геометрическая коса $B^{\prime}$ зафиксирована, мы выберем $\varepsilon>0$. Если $\varepsilon$ достаточно мало, то при любом $t \in[0,1]$ можно предполагать, что $C_{t}^{\prime}$ является $C^{1}$-гладкой окружностью в $D_{t}$, удовлетворяющей тому условию, что $\left(C_{t}^{\prime} \cup B_{t}^{\prime}\right) \cap U_{\varepsilon}(z)$ является связным подмножеством кривой $C_{t}^{\prime}$ при всех $z \in C_{t}^{\prime}$, где $U_{\varepsilon}(z)$ обозначает открытый диск радиуса $\varepsilon$ с центром $z$. Мы можем также предполагать, что $C_{t}^{\prime}$ при всех $t$ имеет минимальную длину среди всех замкнутых кривых своего гомотопического класса, удовлетворяющих этому условию. Тогда каждая кривая $C_{t}^{\prime}$ есть объединение отрезков прямых и дуг окружностей радиусов $\varepsilon, 2 \varepsilon, \ldots$ с центрами в точках множества $B_{t}^{\prime}$.

При каждом $t \in[0,1]$ положим $x_{t}=\min \left\{\operatorname{Re} z \mid(z, t) \in B_{t}^{\prime} \backslash S_{t}^{\prime}\right\}, L_{t}=\left\{(z, t) \in D_{t} \mid\right.$ $\left.\operatorname{Re} z=x_{t}\right\}$ и обозначим через $A_{t}$ нижнюю компоненту связности множества $L_{t} \backslash\left(C_{t}^{\prime} \cap L_{t}\right)$. Пусть $A^{\circ}$ - замькание множества $\bigcup_{t \in[0,1]} A_{t}$. Тогда $\left.p\right|_{A}$ о является локально-тривиальным расслоением всюду, кроме конечного множества точек $t_{1}, \ldots, t_{k}$, каждая из которых расположена вблизи от двойной точки проекции $p\left(B^{\prime} \backslash S^{\prime}\right)$, отвечающей вхождению

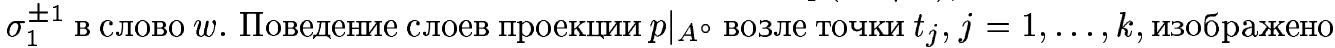
на рис. 2 , где $\delta>0$ (соответственно $\delta<0$ ) отвечает вхождению $\sigma_{1}^{+1}$ (соответственно $\left.\sigma_{1}^{-1}\right)$.

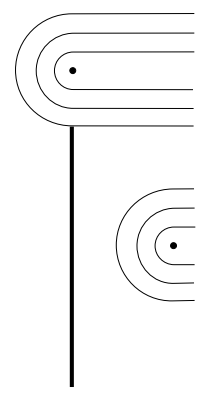

$A_{t_{j}-\delta}$

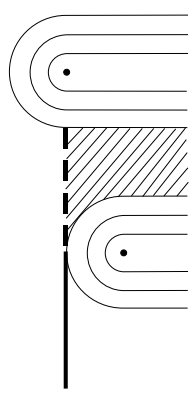

$A_{t_{j}}$

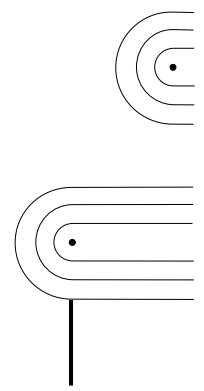

$A_{t_{j}+\delta}$

Рис. 2

Обозначим через $a_{j}$ отрезок $\left(A^{\circ} \cap D_{t_{j}}\right) \backslash A_{t_{j}}$ (изображенный пунктиром на рис. 2). Обозначим через $A_{j}^{*}$ ту компоненту множества $D_{t_{j}} \backslash\left(C_{t_{j}}^{\prime} \cup a_{j}\right)$, которая расположена справа от $a_{j}$ (заштрихована на рис. 2), и пусть $A=A^{\circ} \cup A^{*}$, где $A^{*}=A_{1}^{*} \cup \cdots \cup A_{k}^{*}$.

Вычислим индекс зацепления $S^{\prime}$ и $C^{\prime}$. Он равен индексу пересечения $S^{\prime}$ и $A$. Мы имеем $S^{\prime} \cap A=\left(S^{\prime} \cap A^{\circ}\right) \cup\left(S^{\prime} \cap A^{*}\right)$. Точки из $S^{\prime} \cap A^{\circ}$ отвечают тем вхождениям $\sigma_{1}^{s}, s= \pm 1$, в слово $w$, для которых соответствующая двойная точка проекции $p\left(B^{\prime}\right)$ является точкой пересечения кривых $p\left(S^{\prime}\right)$ и $p\left(B^{\prime} \backslash S^{\prime}\right)$. Точки из $S^{\prime} \cap A^{*}$ отвечают тем вхождениям $\sigma_{1}^{s}$, $s= \pm 1$, в слово $w$, которые соответствуют двойным точкам проекции $p\left(B^{\prime} \backslash S^{\prime}\right)$ (заметим, что каждое $A_{j}^{*}$ должно содержать точку из $S^{\prime} \cap D_{t_{j}}$, потому что $C^{\prime}$ минимальна). В обоих случаях вклад точки пересечения в индекс зацепления равен $s$. Поскольку индекс зацепления равен +1 , мы видим, что любое слово, представляющее $\beta^{\prime}$, должно включать в себя $\sigma_{1}^{+1}$. Теорема В доказана.

2. Геометрическая интерпретация право-инвариантного порядка. Все отображения, рассматриваемые ниже, будут кусочно гладкими. Пусть $D$ и $X_{n}=\left\{x_{0}, x_{1}\right.$, $\left.\ldots, x_{n+1}\right\}$ - обозначают то же, что и в п. 1 . Группу кос $B_{n}$ можно естественно отождествить с группой классов отображений пары $\left(D, X_{n}\right)$, которая по определению есть 
группа гомеоморфизмов $\left(D, X_{n}\right) \rightarrow\left(D, X_{n}\right)$, тождественньх на $\partial D$ с точностью до гомеоморфизмов, изотопных тождественному отображению. Класс $[\gamma] \in B_{n}$ гомеоморфизма $\gamma$ однозначно определен кривой $\Gamma:=\gamma([-1,1])$. Следуя [4], назовем $\Gamma$ криволинейной диаграммой (curve diagram) косы $[\gamma]$. Криволинейная диаграмма косы $\sigma_{i}$ имеет вид $\left[-1, x_{i-1}\right] \cup \Gamma_{+} \cup\left[x_{i}, x_{i+1}\right] \cup \Gamma_{-} \cup\left[x_{i+2}, 1\right]$, где $\Gamma_{+}-$путь из $x_{i-1}$ в $x_{i+1}$ по верхнему полудиску и $\Gamma_{-}-$путь из $x_{i}$ в $x_{i+2}$ по нижнему полудиску. Соглашение о порядке умножения проиллюстрировано на рис. 3 (мы воспроизводим его из [4]).

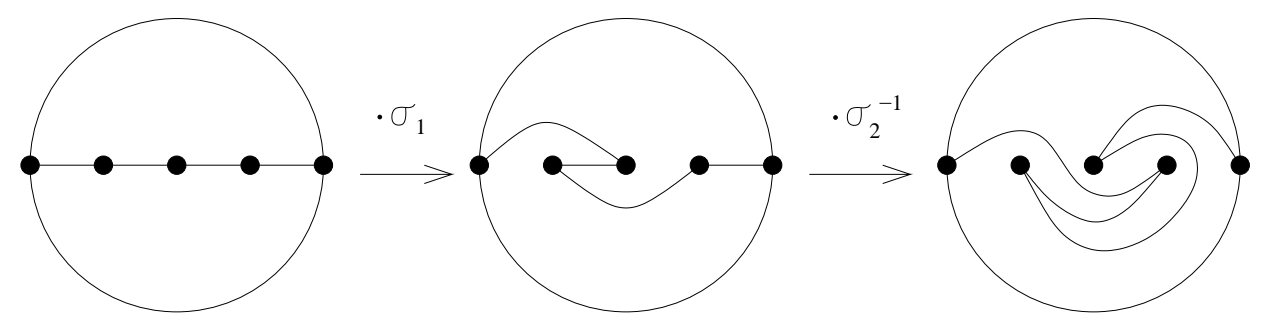

Рис. 3. Криволинейные диаграммы кос $1, \sigma_{1}$ и $\sigma_{1} \sigma_{2}^{-1}$

Пусть $\Gamma=\gamma([-1,1])-$ криволинейная диаграмма. Обозначим $\gamma\left(\left[x_{i}, x_{i+1}\right]\right)$ через $\Gamma_{i}$. Будем говорить, что криволинейная диаграмма $\Delta$ трансверсальна к $\Gamma$, если для каждой $\Gamma_{i}$ либо $\Gamma_{i} \subset \Delta$, либо $\Gamma_{i}$ трансверсальна к $\Delta$.

Пусть $\Delta$ трансверсальна к $\Gamma$. Компонента множества $D \backslash(\Gamma \cup \Delta)$ называется двуугольником межсду Г и $\Delta$, если она гомеоморфна открытому диску и ограничена одним открытым отрезком кривой $\Gamma \backslash X_{n}$, одним открытьм отрезком кривой $\Delta \backslash X_{n}$ и двумя точками. Будем говорить, что пара $(\Gamma, \Delta)$ редуцирована, если нет двуугольников между Г и $\Delta$.
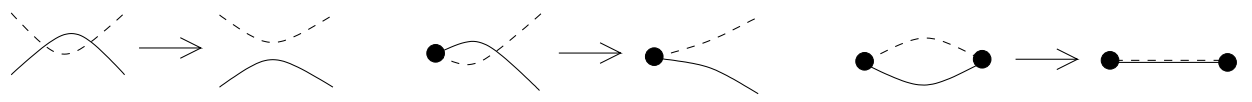

Рис. 4

Любую пару криволинейных диаграмм $(\Gamma, \Delta)$ можно редуцировать за конечное число элементарных преобразований, изображенных на рис. 4 , где Г изображена сплошной линией, а $\Delta$ - пунктирной. Каждое элементарное преобразование уменьшает на 1 число двуугольников.

Право-инвариантный порядок на группе кос можно описать в терминах криволинейных диаграмм следующим образом (см. [4]). Пусть косы $\gamma, \delta$ заданы трансверсальньми друг другу криволинейными диаграммами $\Gamma, \Delta$. Предположим, что $(\Gamma, \Delta)$ редуцирована. Множество $D \backslash \Gamma$ состоит из двух компонент. Та компонента, которая содержит $\sqrt{-1}$, назьвается верхней. Предположим, что $Г$ и $\Delta$ совпадают на дуге, идущей из -1 в $x_{i}$ (возможно, $i=0)$, и не совпадают после $x_{i}$. Пусть $a-$ компонента множества $\Delta \backslash(\Delta \cap \Gamma)$, начинающаяся в $x_{i}$. Тогда $\delta>\gamma$, если и только если $a$ лежит в верхней части $D \backslash \Gamma$. Это значит, что двигаясь вдоль $\Delta$ из -1 в 1 , мы идем вдоль $Г$ до точки $x_{i}$, а затем сворачиваем влево. 
3. Доказательство теоремы А. Поскольку все образующие сопряжены между собой, достаточно доказать, что $\gamma \sigma_{1} \gamma^{-1}>1$ для всех $\gamma \in B_{n}$. В силу право-инвариантности это равносильно тому, что $\gamma \sigma_{1}>\gamma$.

Пусть $\gamma$-произвольная коса из $B_{n}$. Нам надо доказать, что $\delta>\gamma$, где $\delta=\gamma \sigma_{1}$. Пусть $\Gamma$ - криволинейная диаграмма косы $\gamma$. Пусть $I=[-1,1]$, это криволинейная диаграмма единичной косы. Предположим, что $\Gamma$ трансверсальна к $I$ и пара $(\Gamma, I)$ редуцирована. Обозначим $\varepsilon$-окрестность отрезка $\left[x_{1}, x_{2}\right]$ через $D_{\varepsilon}$. Поскольку $(\Gamma, I)$ редуцирована, при достаточно малом $\varepsilon$ пара $\left(D_{\varepsilon}, D_{\varepsilon} \cap \Gamma\right)$ изотопна одной из нарисованных на рис. 5 и выполнено следуюшее условие:

$(*)$ если две точки из $\Gamma \cap \partial D_{\varepsilon}$ соседние в смысле циклического порядка вдоль окружности $\partial D_{\varepsilon}$, то их нельзя соединить дугой из $\Gamma \backslash D_{\varepsilon}$, не содержащей точек из $X_{n}$.

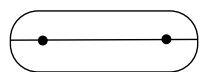

A

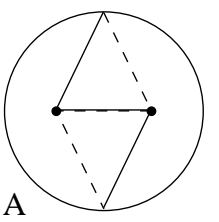

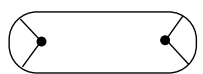

$\mathrm{B}_{0}$

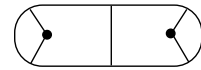

$\mathrm{B}_{1}$

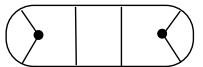

$\mathrm{B}_{2}$

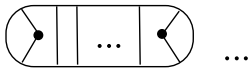

$\mathrm{B}_{\mathrm{k}}$

Рис .5
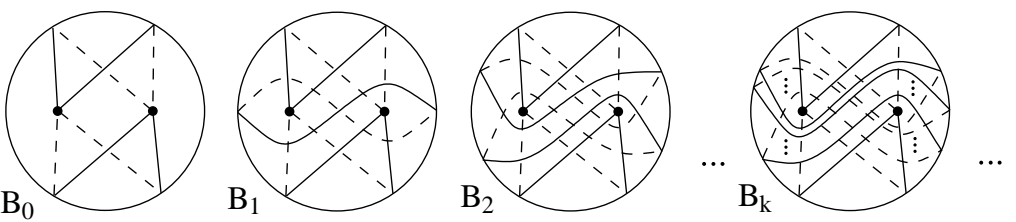

РиС. 6

Кривые $D_{\varepsilon} \cap \Gamma$ на рис. 5 можно продеформировать в кривые, изображенные слошной линией на рис. 6 . Криволинейная диаграмма $\Delta_{\varepsilon}$ косы $\delta:=\gamma \sigma_{1}$ получается из Г заменой фрагмента $D_{\varepsilon} \cap \Gamma$ на кривые, изображенные на рис. 6 пунктиром. Диаграмма $\Delta_{\varepsilon}$ еше не трансверсальна к $\Gamma$. Пусть $\Delta$ получена из $\Delta_{\varepsilon}$ применением преобразования, показанного на рис. 7 , в каждой из тех компонент множества $\Gamma \backslash\left(D_{\varepsilon} \cup X_{n}\right)$, у которых один из концов лежит на $\partial D_{\varepsilon}$. Диаграмма $\Delta$ уже трансверсальна к $\Gamma$.
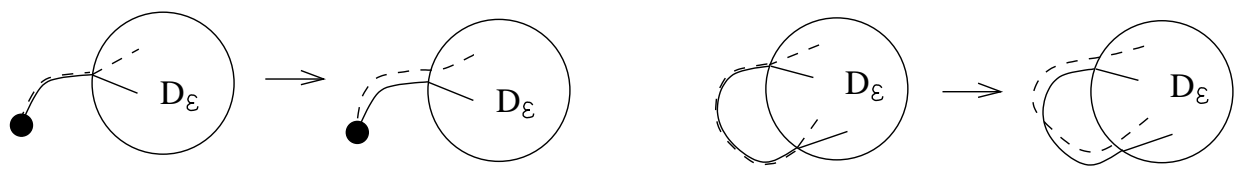

Рис. 7

Если имеет место случай, показанный на рис. $5 . \mathrm{A}$, то $(\Gamma, \Delta)$ редуцирована и требуемое неравенство $\delta>\gamma$ непосредственно вытекает из результата [4], сформулированного в п. 1. Поэтому мы будем предполагать, что имеет место один из случаев рис. $5 . \mathrm{B}_{k}$.

$\Gamma \cup \Delta$ разбивает $D_{\varepsilon}$ на несколько криволинейных многоугольников. Среди них $2 k+2$ треугольников и $2 k+2$ пятиугольников (четырехугольников при $k=0$ ) примькают к окружности $\partial D_{\varepsilon}$. Обозначим их соответственно через $T_{i}, P_{i}, i=1, \ldots, 2 k+2$. Скажем, 
что треугольник $T_{i}$ плохой, если он получился из треугольника на рис. 6, у которого стороны, лежащие на $\partial D_{\varepsilon}$, соединены вне $D_{\varepsilon}$ дугой диаграммы $\Gamma$. По $(*)$ эта дуга должна содержать точку из $X_{n}$. Присоединяя область, ограниченную этой дугой, к плохому треугольнику, мы получаем двуугольник между $\Gamma$ и $\Delta$, причем все двуугольники между $\Gamma$ и $\Delta$ получаются таким способом.
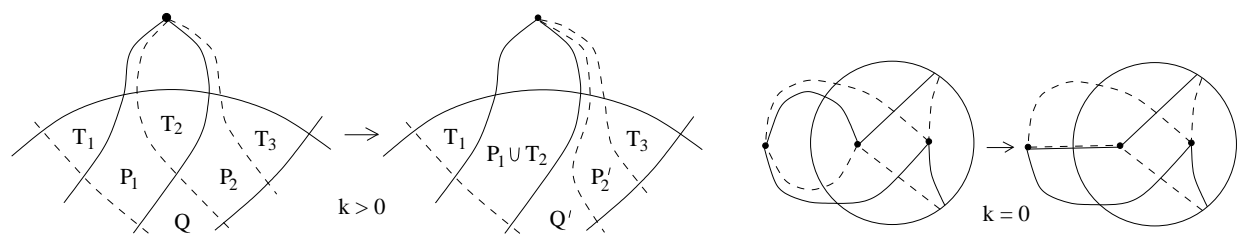

Рис. 8

Пусть $\Delta^{\prime}$ получена из $\Delta$ применением преобразования, показанного на рис. 8, к каждому плохому треугольнику. Поскольку плохое треугольники не могут быть соседними, эти преобразования независимы. Покажем, что $\left(\Gamma, \Delta^{\prime}\right)$ редуцирована. Действительно, при $k=0$ это очевидно (см. рис. 8). При $k>0$ появляется только три новых многоугольника: $Q^{\prime}, P_{2}^{\prime}$, и $P_{1} \cup T_{2}$ (см. рис. 8 ). Ни один из них не может содержаться в двуугольнике между $Г$ и $\Delta^{\prime}$.

Мы видим на рис. 8 , что $\Delta^{\prime}$ была получена из $\Delta$ изменением только тех отрезков $\Delta, \mathrm{y}$ которых оба конца лежат в $D_{\varepsilon}$. Однако точка $x_{i}$, в которой $\Delta^{\prime}$ впервые расходится с $\Gamma$, лежит на отрезке, начинающемся в $x_{0}$ и не входящем в $D_{\varepsilon}$. Следовательно, $\Delta^{\prime}$ совпадает с $\Delta$ возле $x_{i}$. Но возле $x_{i}$ кривая $\Delta$ расположена относительно $\Gamma$, как на рис. 7 . Значит, $\delta>\gamma$ согласно п. 1. Теорема А доказана.

\section{СПИСОК ЦИТИРОВАННОЙ ЛИТЕРАТУРЫ}

[1] Dehornoy P. Braid groups and left distributive operations // Trans. Amer. Math. Soc. 1994. V. 343. P. 115-150.

[2] Dehornoy P. From large cardinals to braids via distributive algebra // J. Knot Theory and Its Ramifications. 1995. V. 4. P. 33-79.

[3] Dehornoy P. A fast method of comparing braids // Adv. in Math. 1997. V. 125. P. 200-235.

[4] Fenn R., Greene M. T., Rolfsen D., Rourke C., Wiest B. Ordering the braid group // Pacific J. Math. 1999. V. 191. P. 49-74.

[5] Burckel S. The well-ordering on positive braids // J. Pure Appl. Algebra. 1997. V. 120. P. 1-17.

[6] Laver R. Braid group actions on left distributive structures and well-orderings in the braid groups // J. Pure Appl. Algebra. 1996. V. 108. P. 81-98.

[7] Wiest B. Dehornoy's ordering of the braid groups extends the subword ordering // Pacific J. Math. 1999. V. 191. P. 183-188.

[8] Rudolph L. Algebraic functions and closed braids // Topology. 1983. V. 22. P. 191-202.

[9] Rudolph L. Some knot theory of complex plane curves // Noeuds, Tresses et Singularités. Mono. № 31, L’Ens. Math., 1983. P. 99-122.

[10] Orevkov S. Yu. Link theory and oval arrangements of real algebraic curves // Topology. 1999. V. 38. P. $779-810$.

Математический институт им. В. А. Стеклова РАН

Поступило

E-mail: orevkov@mi.ras.ru

30.12.1998 